\title{
Cuidados alternativos en la atención del embarazo en Jalisco, México
}

\author{
Alternative care options for pregnancy \\ in Jalisco, Mexico
}

Isabel Valadez 1

Noe Alfaro 1

Elizabeth Pozos 1

1 Instituto Regional de Investigación en Salud Pública, Centro Universitario de Ciencias de la Salud, Universidad de Guadalajara. Francisco Labastida 653, Código Postal 44290,

Guadalajara, Jalisco, México. dravaladez@hotmail.com dravaladez@megared.net.mx

\begin{abstract}
The mother's culture has been identified as a factor in the use of health services for prenatal care. Beliefs and practices concerning traditional and domestic medicine used by women during pregnancy can play a role in the decision to seek medical care. In a non-probabilistic sample of 3,250 women, alternative care during pregnancy and its relationship to satisfactory prenatal care was studied. To analyze the association, the prenatal care variable was divided into (a) satisfactory and (b) unsatisfactory, according to a list of official Mexican standards. The chisquare test and odds ratio were applied, with $p \leq 0.05$. The association of alternative care with unsatisfactory prenatal care was established. Psycho-prophylactic training for delivery acted as a protective factor. The culture of pregnant woman in Mexico comprises meaning and behaviors that mix concepts and prescriptive ideas concerning domestic, traditional, and academic medicine, varying according to the stage of pregnancy, and which may be used in exclusive or complementary fashion. This situation should be considered in health education programs.
\end{abstract}

Key words Prenatal Care; Alternative Care; Pregnancy; Health Services

Resumen En la atención prenatal se ha identificado la cultura de la madre como factor que influye en la utilización de los servicios de salud. Las creencias y prácticas de medicina tradicional y doméstica que realiza la mujer durante el embarazo pueden intervenir en la decisión de acudir a la atención médica. En una muestra no probabilística de 3.250 mujeres se identificaron los cuidados alternativos de atención al embarazo y se estableció su relación con un control prenatal satisfactorio. Para el análisis de asociación se dividió la variable de atención prenatal en (a) satisfactoria y (b) no satisfactoria de acuerdo a la Norma Oficial Mexicana. Se empleó la prueba de chi ${ }^{2}$, el odds ratio y sus intervalos de confianza. Los cuidados alternativos resultaron asociados a una atención prenatal no satisfactoria. El entrenamiento psicoprofiláctico se comportó como factor protector. La cultura de la gestante es una configuración de significados y comportamientos, con mezcla de concepciones y prescripciones de la medicina doméstica, tradicional y académica, que varían según la etapa del embarazo, que pueden ser únicas o complementarias. Esta situación debe considerarse en los programas educativos en salud.

Palabras claves Atención Prenatal; Cuidados Alternativos; Embarazo; Servicios de Salud 


\section{Introducción}

Entre las acciones emprendidas para mejorar la salud de la madre y del hijo se encuentran las concernientes al cuidado del embarazo. La prestación de servicios institucionales de cuidado y control prenatal han hecho un aporte importante a la disminución de los índices de morbilidad y mortalidad materno infantil. Sin embargo, la sola presencia de estos servicios no garantiza su utilización oportuna y con la frecuencia requerida. Existen factores que afectan su utilización, ya sea animándola o inhibiéndola. En los estudios realizados sobre la utilización de la atención prenatal, se han identificado factores maternos, dentro de los cuales se mencionan las cuestiones culturales alrededor del embarazo y su atención, que definen la actuación de la mujer gestante (Blondell \& Marshall, 1998; Infante, 1990). Por ello, si se emplea un criterio más amplio para definir los conceptos de vigilancia y control del embarazo, se puede reconocer un buen número de acciones destinadas a cuidar la salud de la madre, que comprenden, además del sector salud, el sistema social en general.

En México, la existencia de un "sistema real de salud" está integrado por tres modelos: medicina académica o institucional, medicina tradicional y medicina doméstica. Estas dos últimas no están institucionalizadas, pero están estructuradas, tienen contradicciones con la medicina académica, pero interactúan con ella. En la medicina tradicional, los médicos tradicionales se ocupan de síndromes de filiación cultural (susto, espanto, empacho, tirantez o quebradura de espíritu), que no se encuentran en los libros de medicina ortodoxa. En estas y otras enfermedades no tradicionales, conciben, previenen, diagnostican, realizan el tratamiento y hacen el pronóstico de acuerdo a claves profundas de la cultura del grupo (Menéndez, 1990; Zolla, 1987; Zolla \& Carrillo, 1998). La persistencia de la medicina tradicional se ha explicado por la existencia de una estructura común de pensamiento, además de por la importancia que proporciona a aspectos psíquicos y el hecho de que considere al paciente como un todo interrelacionado con el cuerpo social y con el universo (Martínez, 1992; Mellado et al., 1989). El único terapeuta tradicional tolerado en la práctica por el estado y la medicina académica es la partera, a la que se considera indispensable capacitar. En México hay muchísimas mujeres que prefieren a las parteras empíricas, aún cuando cuentan con seguridad social y hayan sido atendidas en alguna ocasión por profesionales de la salud (SSA, 1989). El modelo de atención de la medicina doméstica tiene como escenario importante el núcleo familiar, con la autoatención como forma concreta. Es por ello que Carlos Zolla (Zolla \& Carrillo, 1988) en sus investigaciones la denomine "medicina doméstica”. Está estructurada como un sistema de conceptos, creencias y prácticas relativas a los estados de desequilibrio en general y al proceso salud enfermedad en particular. Simultáneamente es una estructura de servicios y un espacio de toma de decisiones, relativos a la atención casera o a la canalización del enfermo hacia otros sistemas médicos. La atención casera y/o la canalización están basadas en estimaciones de carácter clínico, oferta y demanda, prestigio, creencias y respuesta a lo que oyen, ya sea de otros miembros del grupo o de las instituciones. $\mathrm{Al}$ relacionarse con la medicina académica y con la medicina tradicional, para la atención del enfermo o enfermos dentro del grupo doméstico, se sitúa como agente de vinculación y síntesis. Lo que explica en buena medida la hibridez de muchos de los conocimientos que posee y de las prácticas que realiza. De acuerdo con Menéndez (1990), la perspectiva estructural de la medicina doméstica considera la autoatención centrada en la mujer el primer nivel de atención. En México, se tienen datos de la existencia de un modelo mixto de atención en ginecoobstetricia (León, 1905), que se mantiene hasta el presente, dado el carácter de formación socioeconómica mexicana (García, 2000).

En el contexto de que para lograr el cumplimiento de los objetivos de atención prenatal es necesario, por un lado asegurar la cobertura de los servicios, y por el otro que la mujer acuda a demandar el servicio en fechas tempranas de la gestación y con la periodicidad necesaria, partimos en este estudio de que las prácticas de cuidado alternativo en la atención del embarazo, que llevan a cabo las mujeres gestantes, pueden comportarse como un factor de riesgo o constituir parte de la cadena causal de hechos y situaciones que influyen no sólo en la decisión de acudir a la atención prenatal. Para dar respuesta a nuestros supuestos y establecer cuál es el comportamiento de los cuidados alternativos en la utilización de los servicios institucionales de atención prenatal, se diseñó un estudio de corte cuantitativo, que permitiera en un primer momento identificar los cuidados alternativos que llevan a cabo las embarazadas, $\mathrm{y}$ en un segundo momento, establecer su asociación con la demanda oportuna de la atención prenatal. 


\section{Material y método}

Se estudió una muestra no probabilística de 3.250 mujeres en puerperio de bajo riesgo, o de egreso hospitalario temprano (IMSS, 1983; SSA, 1983), en hospitales de atención obstétrica de la Zona Metropolitana de Guadalajara y de 12 Hospitales Regionales de Zona ubicados en localidades del interior del Estado de Jalisco; pertenecientes al Sector Salud: Instituto Mexicano del Seguro Social (IMSS); Instituto de Seguridad Social al Servicio de los Trabajadores del Estado (ISSSTE); Secretaría de la Defensa Nacional (SEDENA) y Secretaría de Salud Jalisco (SSJ) (Figura 1).

La muestra se dividió proporcionalmente para cada zona de estudio. Se utilizó muestreo en dos etapas estratificado por conglomerados para la selección de los casos; la variable de estratificación fue la seguridad social, tomando como primera etapa de selección la zona de ubicación de los Hospitales de estudio (Zona Metropolitana de Guadalajara y localidades del interior del Estado). La segunda etapa fue en el interior de cada unidad seleccionando aleatoriamente a las mujeres en base a los listados diarios de nacimientos (Silva, 1990).

Se utilizó un formulario estructurado, que incluyó datos generales de la mujer, nivel económico social (Germani \& Ferrera, 1985), que involucra en su medición tres parámetros: (1) educación, (2) ocupación del jefe de familia y (3) el ingreso familiar; antecedentes ginecoobstétricos y oportunidad de la atención médica. El formulario constó, además, de un apartado que indagó sobre la realización de prácticas de cuidados alternativos de atención al embarazo.

La información se analizó utilizando frecuencias absolutas y relativas, y medidas de tendencia central, según el tipo de variables. Para determinar la asociación de los cuidados alternativos al embarazo con la atención prenatal, se clasificó a las mujeres de acuerdo al modelo establecido en las Normas Oficiales Mexicanas del Sector Salud (NOMSS) para la atención de la mujer durante el embarazo, parto y puerperio y del recién nacido (SSA, 1994), considerado en un informe reciente por la Organización Panamericana de la Salud como un modelo adecuado (Villar \& Bergsjo, 1997) en las siguientes categorías:

(1) Atención prenatal satisfactoria - cuando la mujer inició la atención prenatal en el transcurso de las primeras 12 semanas de gestación, recibió 5 o más consultas, y estas fueron en las semanas de gestación referidas por las NOMSS.

(2) Atención prenatal no satisfactoria - cuando la mujer inició la atención prenatal después de las primeras 12 semanas de gestación; o bien cuando habiendo iniciado la atención prenatal en las primeras 12 semanas de gestación, no acumuló el número de consultas establecidas para las semanas de gestación

(3) No atención prenatal - cuando no recibieron ninguna consulta médica durante su embarazo.

Se manejaron tablas de contingencia 2 X 2 y se empleó la ji ${ }^{2}$, el odds ratio (OR) y sus intervalos de confianza, para identificar su asociación con dicho control prenatal; Se consideró como tal cuando la ji2 resultó mayor de 3,84 $(\mathrm{P}<0,05)$ y cuando el OR fue mayor de 1 y que el intervalo de confianza no incluyera la unidad.

Figura 1

Universo de estudio. Ubicación geográfica de los hospitales de atención obstétrica en Jalisco, México.
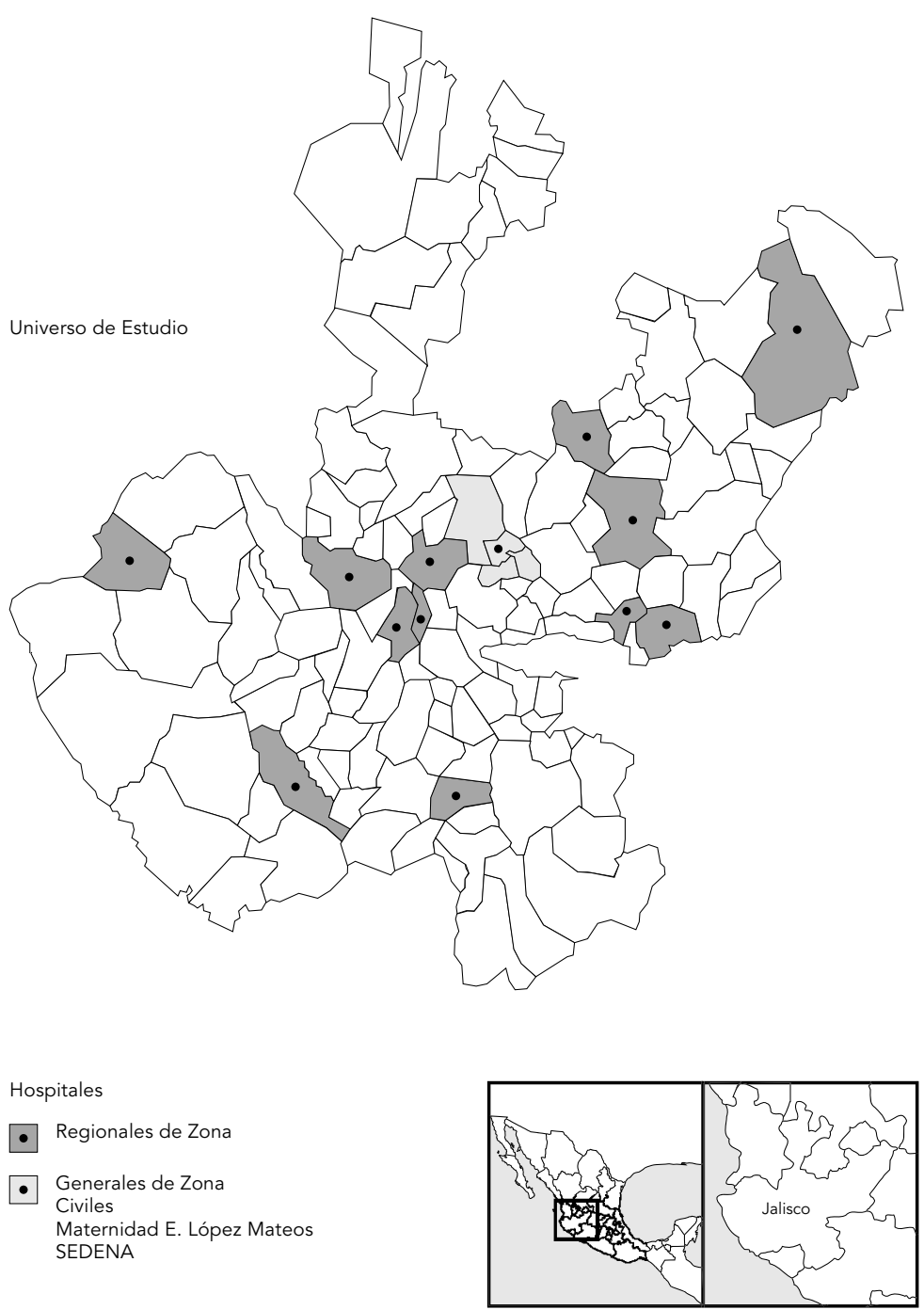

Cad. Saúde Pública, Rio de Janeiro, 19(5):1313-1321, set-out, 2003 


\section{Resultados}

En la realización de la entrevista no se presentó rechazo o interrupción de la misma y se logró estudiar un total de 3.250 mujeres. El 50,0\% de las mujeres entrevistadas fueron de hospitales de la Zona Metropolitana de Guadalajara; y el otro 50,0 \% correspondieron a Hospitales Regionales del interior del Estado de Jalisco. Las características de la población de estudio se señalan en la Tabla 1. Se destaca la presencia de una proporción de mujeres fuera de los límites de edad marcados como óptimos y una paridad alta que rebasa el límite crítico de cuatro hijos por mujer (Langer \& Lozano, 1998).

\section{Atención alternativa al embarazo}

Los cuidados alternativos fueron mencionados en un $47,7 \%$ de las mujeres estudiadas, la distribución de acuerdo a la atención prenatal se muestra en la Tabla 2. Los cuidados alternativos mencionados fueron la utilización de remedio caseros; la supresión de alimentos de la dieta; el acomodo del producto y el entrenamiento para parto psicoprofiláctico.

De acuerdo con los resultados obtenidos, el $23,2 \%$ (754 mujeres) tomaron remedios caseros, estos se dividieron en remedios utilizados para molestias de orden general y los específi-

Tabla 1

Características de las mujeres del estudio.

Jalisco, México.

\begin{tabular}{|c|c|c|}
\hline Característica & $n^{*}$ & $\%$ \\
\hline \multicolumn{3}{|c|}{ Nivel económico social } \\
\hline Alto & 597 & 18,3 \\
\hline Medio & 889 & 27,5 \\
\hline Bajo & 1.764 & 54,0 \\
\hline \multicolumn{3}{|l|}{ Edad (años) } \\
\hline$>19$ & 575 & 17,5 \\
\hline $20-29$ & 904 & 58,5 \\
\hline$\geq 30$ & 782 & 24,0 \\
\hline Promedio & $25,4 \pm 5,98$ & \\
\hline \multicolumn{3}{|c|}{ Número de embarazos } \\
\hline Primigestas & 998 & 30,5 \\
\hline $2-3$ & 361 & 41,8 \\
\hline $4-6$ & 705 & 21,7 \\
\hline$\geq 7$ & 186 & 5,5 \\
\hline Promedio & $2,8 \pm 2$ & \\
\hline
\end{tabular}

${ }^{\star} \mathrm{n}=3.250$. cos relacionados con las molestias del embarazo, tales como estados nauseosos. Los primeros fueron igualados a la ingestión de infusiones o té de plantas, dentro de los cuales nos refirieron en orden de frecuencia: la manzanilla, el romero, la hierbabuena, el té de las siete flores, la albaca, el comino, el limón, el orégano, las hojas de guayaba, té de maíz, la ruda, las flores de nochebuena y el poleo, las cuales fueron ingeridas solas o en combinación. La infusión más usada fue la manzanilla, cuya ingesta se realizó durante toda la gestación, sin embargo, entre sus funciones mencionadas está el tonificar el útero, regulador de las menstruaciones. La manzanilla contiene proazulenos, terpenos, flavónicos, taninos, alcanfor, y cumarinas, sustancias de reconocidos efectos antiinflamatorios, antisépticos y antiespasmódicos, y que en algunas literaturas se recomienda su uso moderado durante el embarazo, y en otras se menciona como contraindicada su uso durante el embarazo (Argueta \& Cano, 1994; Jaramillo, 1998; Lara \& Márquez, 1996). Los motivos de ingesta fueron referidos principalmente por trastornos y afecciones estomacales como flatulencia, mala digestión, insomnio y nerviosismo. Le siguió en orden de frecuencia, el romero, el cual es rico en ácido úrlico, alcanfor taninos amargos, cuyas características más importantes son funcionar como antiinflamatorio y analgésico. No encontrándose mención en la literatura de precauciones. La ruda que tiene capacidad estrogénica debido a ciertos isoflavonas lipofilas, además, contiene rutina, cumarina, cetonas y flavonoides, contraindicadas durante el embarazo, con funciones de tonificar y calentar el útero e induce las menstruaciones. Las flores de nochebuena fueron específicamente empleadas en el primer trimestre del embarazo para confirmar el embarazo, la ruda se utilizó además en el último trimestre como coadyuvante en el trabajo de parto, (Argueta, 1994; Jaramillo, 1998; Lara \& Márquez, 1996). El té de poleo que contiene entre otros taninos pulegonas cipentanos, cuya utilización fue mencionada como caliente para "aflojar" los huesos de la cadera, como preparación para el parto. Se menciona en sus funciones la de calentar el útero, y promover las menstruaciones, por lo que se contraindica su uso en el embarazo. Es importante aclarar que una misma planta fue utilizada para el manejo de varios problemas y que hay plantas a las cuales se les atribuyó poderes curativos muy amplios (Argueta, 1994; Jaramillo, 1998; Lara \& Márquez, 1996). Dentro de los remedios específicos relacionados con los trastornos del embarazo se mencionó su utilización, principalmente en el 


\begin{tabular}{|c|c|c|c|c|c|c|c|c|}
\hline \multirow[t]{2}{*}{ Característica } & \multicolumn{2}{|c|}{$\begin{array}{l}\text { Utilización de } \\
\text { remedios caseros }\end{array}$} & \multicolumn{2}{|c|}{$\begin{array}{l}\text { Supresión } \\
\text { dealimentos }\end{array}$} & \multicolumn{2}{|c|}{$\begin{array}{l}\text { Acomodo } \\
\text { del niño }\end{array}$} & \multicolumn{2}{|c|}{$\begin{array}{l}\text { Entrenamiento parto } \\
\text { psicoprofiláctico }\end{array}$} \\
\hline & $n^{*}$ & $\%$ & $n^{*}$ & $\%$ & $n^{*}$ & $\%$ & $n^{*}$ & $\%$ \\
\hline \multicolumn{9}{|l|}{ Zona de estudio } \\
\hline ZMG & 357 & 47,3 & 457 & 70,2 & 505 & 59,8 & 65 & 32,0 \\
\hline Interior del Estado & 397 & 2,7 & 194 & 29,8 & 339 & 40,2 & 138 & 68,0 \\
\hline \multicolumn{9}{|l|}{ Nivel económico social } \\
\hline Alto & 123 & 16,4 & 123 & 18,9 & 115 & 13,5 & 61 & 30,0 \\
\hline Medio & 270 & 35,8 & 216 & 33,2 & 265 & 31,4 & 45 & 22,2 \\
\hline Bajo & 361 & 47,8 & 312 & 47,9 & 464 & 54,9 & 97 & 47,8 \\
\hline \multicolumn{9}{|c|}{ Edad de la madre (años) } \\
\hline$<19$ & 92 & 12,2 & 111 & 17,0 & 175 & 20,7 & 39 & 19,3 \\
\hline $20-29$ & 466 & 61,8 & 367 & 56,4 & 478 & 56,7 & 116 & 57,2 \\
\hline$\geq 30$ & 196 & 26,0 & 173 & 26,6 & 191 & 22,7 & 48 & 23,5 \\
\hline \multicolumn{9}{|l|}{ Número de embarazos } \\
\hline Primigesta & 48 & 6,4 & 80 & 12,3 & 68 & 8,0 & 80 & 12,3 \\
\hline $1-3$ & 520 & 69,0 & 431 & 66,2 & 574 & 68,1 & 431 & 66,0 \\
\hline$\geq 4$ & 186 & 24,6 & 140 & 21,1 & 202 & 3,9 & 140 & 21,5 \\
\hline \multicolumn{9}{|l|}{ Periodo de gestación } \\
\hline 1ㅇ Trimestre & 128 & 17,0 & 340 & 52,1 & 23 & 2,7 & 340 & 52,1 \\
\hline 2o Trimestre & 254 & 33,6 & 218 & 33,6 & 141 & 16,7 & 218 & 33,6 \\
\hline 3o Trimestre & 372 & 49,4 & 93 & 14,3 & 680 & 80,6 & 93 & 14,3 \\
\hline \multicolumn{9}{|c|}{ Atención médica embarazo } \\
\hline Satisfactoria & 500 & 66,3 & 435 & 66,8 & 520 & 63,2 & 435 & 66,8 \\
\hline No satisfactoria & 208 & 27,6 & 173 & 26,5 & 209 & 25,3 & 173 & 26,5 \\
\hline No atención & 46 & 6,1 & 43 & 7,0 & 96 & 11,3 & 43 & 70,0 \\
\hline Subgrupos & 754 & 23,2 & 651 & 20,0 & 822 & 25,1 & 203 & 6,2 \\
\hline
\end{tabular}

${ }^{*} \mathrm{n}$ total $=3.250$.

primer trimestre del mismo, para "cortar" los ascos, las frutas ácidas como el mango, limón pepino y jícama condimentados con limón y sal. Preparados a base de combinaciones de verduras y frutas tales como el betabel, piña y apio con jugo de limón. También, dentro de la medicina de patente, el suero oral como preparación del estómago para el resto de los alimentos o como alimento único, dependiendo de la severidad de las molestias. La mayoría de los remedios fueron administrados a iniciativa de la gestante en un segundo lugar por los familiares, siguiendo consejos de amigos y médicos, dentro de estos últimos, la manzanilla y el té de limón fueron los recomendados. Esta situación se presentó por igual en ambas zonas de estudio, hospitales de la zona metropolitana de Guadalajara, y hospitales del interior del esta- do, en los niveles económicos medio y bajo, en cualquier etapa del embarazo, y mayoritariamente en mujeres que tuvieron una atención prenatal satisfactoria.

De las 3.250 mujeres del estudio, $651(20,0 \%)$ suprimieron de su dieta habitual alimentos de origen animal (carne de cerdo y mariscos), las vísceras, el huevo y productos lácteos, alimentos considerados como pesados y de difícil digestión. Las legumbres, cereales, y algunas frutas y verduras. Los irritantes como el chile y los condimentos. Entre las causas por las cuales las mujeres suprimieron dichos alimentos se encontró que mencionaron prioritariamente trastornos del embarazo. Este hecho de supresión de alguno o más alimentos se prolongó en algunas de ellas hasta el segundo trimestre y en algunas mujeres durante todo el embarazo. 
El acomodo del niño, realizado por parteras y familiares, se presentó en 822 mujeres $(25,1 \%)$. Utilizado en los primeros meses de la gestación como forma de "desprender el huevo" y en el último mes para evitar que se "pegue el niño" y arreglarlo para que salga bien cuando esta volteado. El entrenamiento para parto psicoprofiláctico se llevó a cabo en 203 gestantes $(6,2 \%)$ del total de las mujeres.

El comportamiento de estos cuidados alternativos se señalan en la Tabla 3. La mayoría de las mujeres llevaron a cabo uno o más cuidados alternativos, ya sea al mismo tiempo o variando de acuerdo a la etapa de embarazo, independientemente de la utilización de la atención médica para el embarazo.

\section{Análisis de asociación}

Al clasificar a las mujeres de acuerdo a la NOMSS para la atención de la mujer durante el embarazo parto y puerperio y del recién nacido (SSA, 1994), se encontró en la población estudiada que 2.234 mujeres $(68,7 \%)$ habían tenido una atención prenatal satisfactoria; 721 (22,2\%) se ubicaron en atención prenatal no satisfactoria y como no atención prenatal 295 mujeres. La Tabla 3 muestra cómo se ubican estas categorías con la presencia de cuidados alternativos. Para el análisis de asociación, no se tomó en cuenta a las mujeres ubicadas en la categoría de no atención prenatal, ya que el hecho de no haber recibido ninguna consulta médica durante su embarazo las excluía de este análisis.

La presencia de cuidados alternativos en la población de estudio resultó asociada a una atención prenatal no satisfactoria $(\mathrm{OR}=1,75$; $\mathrm{IC}$ $=1,44-2,03$ ). Al desglosar los componentes de esta variable en las dimensiones exploradas se encontró que la utilización de remedios caseros, la supresión de alimentos, y el acomodo del niño, se comportaron como factores de riesgo para llevar a cabo una atención prenatal satis- factoria. En contraposición al entrenamiento para parto psicoprofiláctico que se comportó como un factor protector para la atención prenatal no satisfactoria (Tabla 4).

\section{Discusión}

Es de importancia aclarar que el haber tomado para la selección de las mujeres el criterio de puerperio de bajo riesgo, designación que gira alrededor de las características del evento obstétrico, tuvo como resultado que dentro de nuestra muestra se encontraron, mujeres que se quedan embarazadas fuera de los límites de edad marcados como óptimos. Aunque nuestra muestra no fue probabilística, porque nuestro interés estuvo centrado en la validez interna más que en la externa (Kleinbaum \& Kupper, 1998), consideramos que las características generales de las mujeres entrevistadas no difieren de las del resto de la población del Estado de Jalisco, en razón de haber sido captadas en los Hospitales Regionales de Zona que por sus características son receptores de usuarias de las Unidades de Primer Nivel.

La atención a la salud de la embarazada comprende procedimientos prácticos que en su conjunto constituyen patrones complejos de atención, con distintas formas de racionalización y justificación. Los cuidados alternativos, llevados a cabo en la atención al embarazo por las mujeres de este estudio, dan cuenta de un modelo mixto de atención, ya que se proyectaron acciones de las que se ejercen en los ámbitos de la medicina doméstica y de la medicina tradicional. Interactuando como opciones de atención con la medicina académica y con la posibilidad de emplearlas simultáneamente. Utilización que tiene para la mujer un sentido de combinación, la cual varía de acuerdo a la etapa de gestación en que se encuentra. La importancia de esta situación radica primordialmente en una confrontación cultural entre la

Tabla 3

Cuidados alternativos y atención prenatal. Jalisco, México.

\begin{tabular}{|c|c|c|c|c|c|c|}
\hline \multirow[t]{2}{*}{$\begin{array}{l}\text { Cuidados } \\
\text { alternativos }\end{array}$} & \multicolumn{2}{|c|}{$\begin{array}{l}\text { Atención prenatal } \\
\text { satisfactoria }\end{array}$} & \multicolumn{2}{|c|}{$\begin{array}{l}\text { Atención prenatal } \\
\text { no satisfactoria }\end{array}$} & \multicolumn{2}{|c|}{$\begin{array}{l}\text { No atención } \\
\text { prenatal }\end{array}$} \\
\hline & $n^{*}$ & $\%$ & $n^{\star}$ & $\%$ & $n *$ & $\%$ \\
\hline Presentes & 930 & 28,6 & 396 & 12,1 & 225 & 6,9 \\
\hline No presentes & 1.304 & 40,1 & 325 & 10,0 & 70 & 2,1 \\
\hline Subgrupos & 2.234 & 68,7 & 721 & 22,2 & 295 & 9,0 \\
\hline
\end{tabular}

* $\mathrm{n}$ total $=3.250$ 


\begin{tabular}{|c|c|c|c|c|}
\hline & $\begin{array}{l}\text { Atención prenatal } \\
\text { no satisfactoria }(n)\end{array}$ & $\begin{array}{l}\text { Atención prenatal } \\
\text { satisfactoria ( } n \text { ) }\end{array}$ & Odds ratio & IC \\
\hline Cuidados alternativos & & & 1,71 & $1,44-2,03$ \\
\hline Presentes & 396 & 930 & & \\
\hline No presentes & 325 & 1.304 & & \\
\hline Remedios caseros & & & 1,42 & $1,16-1,71$ \\
\hline Presentes & 208 & 500 & & \\
\hline No presentes & 513 & 1.734 & & \\
\hline \multicolumn{5}{|l|}{ Supresión de alimentos } \\
\hline Presente & 173 & 435 & 1,31 & $1,06-1,60$ \\
\hline No presente & 548 & 1.799 & & \\
\hline Acomodo del niño & & & 1,35 & $1,11-1,63$ \\
\hline Presente & 209 & 520 & & \\
\hline No presente & 512 & 1.714 & & \\
\hline $\begin{array}{l}\text { Entrenamiento para } \\
\text { parto psicoprofiláctico }\end{array}$ & & & 0,65 & $0,44-0,96$ \\
\hline Presente & 35 & 163 & & \\
\hline No presente & 696 & 2.071 & & \\
\hline
\end{tabular}

Odds Ratio: $\mathrm{p}<0,05 ; \mathrm{n}=2.955$.

cultura médica y la cultura de la mujer gestante. Y en el posible riesgo que representan los cuidados alternativos a la salud de la madre y del niño, partiendo de que la realización de cuidados alternativos conlleva la no utilización de los servicios de salud con la oportunidad y periodicidad requerida. Y que la utilización de remedios caseros sea realizada con un conocimiento escaso e impreciso. O en el mejor de los casos, que el proceso educativo que lleva a cabo la mujer con la involucración de la pareja durante el entrenamiento psicoprofiláctico, sea lo que le lleve a buscar la atención prenatal médica. Si reflexionamos sobre esto, podemos señalar que en la atención al embarazo, los actores (sistema de atención a la salud y gestante) se relacionan en un campo que es el de la salud (Bordieu, 1990) con intereses similares, es decir, la apropiación de los conocimientos que permitan llevar a término en mejores condiciones el embarazo, tanto para la madre como para el niño; pero con diferentes formas de apropiación y desde un marco cultural diferente (Harris, 1996), el de la cultura médica (medicina académica) y el de la cultura de la mujer (en donde entran en juego la medicina doméstica, la medicina tradicional y su propia experiencia). Teniendo en cuenta la complejidad del tér- mino cultura, consideramos necesario precisar su contenido. Se considera cultura al conjunto de significados, expectativas y comportamientos compartidos por un determinado grupo social, que facilitan y ordenan, limitan y potencian, los intercambios sociales, las producciones simbólicas y materiales y las realizaciones individuales y colectivas dentro de un marco espacial y temporal determinado. La cultura es el resultado de la construcción social a lo largo del tiempo, relacionada con las condiciones materiales, sociales y espirituales que dominan un espacio y un tiempo. Se expresa en significados, valores, costumbres, rituales, instituciones y objetos materiales y simbólicos que rodean la vida individual y colectiva de la comunidad (Bruner, 1988; San Martín, 1999). La cultura médica se adquiere por medio de la educación institucional y se considera como verdadera (determinada y legitimada por el conocimiento institucional) y en ella predomina la ritualización de las formas de comportamiento. Da por establecido que la autoridad que le confiere el reconocimiento institucional garantiza la aceptación de sus prescripciones por parte de la embarazada. Para el médico las conductas que prescribe deben ser seguidas al pie de la letra o de lo contrario la atención prenatal no tendría 
éxito. Antepone la supremacía de sus conocimientos sobre otras prácticas como medio preponderante para la atención prenatal. En consecuencia, no sólo ignora las peculiaridades y diferencias que podrían presentarse durante la atención al embarazo, sino que impone conductas (Lotman \& Uspenskij, 1979; Menéndez, 1990). La cultura de la mujer gestante es una configuración de significados y comportamientos, que las mujeres de manera particular han elaborado inducidas por su contexto, en su vida previa al embarazo y paralelo a él, mediante intercambios espontáneos con el medio familiar y social. Es un reflejo de la memoria colectiva de los sectores populares, en donde se mezclan elementos y prácticas médicas no oficiales, calificadas como empíricas, mágicas, precientíficas y científicas de diferentes épocas, que constituyen recursos existentes para enfrentar el proceso salud-enfermedad (Aguirre, 1970; Leis, 1989), que no son exclusivas de regiones apartadas o étnicas, sino que se practica también en las grandes ciudades (Anzurez, 1983), como lo reflejan los resultados obtenidos, en donde la presencia de cuidados alternativos se presentó por igual en ambas zonas de estudio, predominando la supresión de alimentos en la zona metropolitana de Guadalajara.

En la atención al embarazo es en donde se muestra más claramente la presencia de creencias, conceptos y prácticas en las cuales se entrecruzan los tres modelos de atención a la salud, integrando concepciones y prescripciones, que varían de acuerdo con la etapa gestacional en que se encuentre la mujer. Para la gestante, la cultura médica no es ni la verdadera, ni la única que genera salud. Lo que la lleva a escuchar, creer y llevar a cabo diferentes recomendaciones. La atención médica es sólo una forma entre varias de atender el embarazo. La mujer deduce que tiene la libertad de elegir qué tipo de indicaciones y a quién escuchar y cuándo puede realizarlas, porque concibe que existen varias formas de cuidar del embarazo y todas ellas son valiosas. Como es el caso de la utilización de los remedios caseros, como un recurso para resolver situaciones transitorias, con elementos visualizados como naturales, o con lógicas de actuación en donde lo desacomodado (el niño) hay que acomodarlo. Durante su embarazo la mujer realiza evaluaciones constantes de su apariencia física, conforme el curso del mismo, de las interferencias de los síntomas en sus actividades, evalúa y determina qué tipo de atención requiere y en qué momento, de acuerdo a lo que considere. Concediendo importancia si es en los inicios del embarazo o al final del mismo. Tanto las prescrip- ciones médicas o prácticas médicas no oficiales son consideradas con el mismo valor e incluso pueden ser contempladas como únicas o complementarias. En lo que respecta a los posibles riesgos a la salud de la madre y del niño, de acuerdo a los resultados obtenidos, el recurso más utilizado en la categoría de remedios caseros fue la herbolaria, presentando un patrón homogéneo. Muchas de las plantas empleadas por estas mujeres, constituyen vegetales prescritos con mucha frecuencia en la medicina tradicional para atender trastornos en la esfera de la reproducción femenina. El uso de una misma planta para varias molestias e indistintamente del período de gestación, así como las combinaciones realizadas, puede deberse a que la mujer tiene un conocimiento escaso o impreciso respecto a sus contraindicaciones y precauciones. Aunque en este estudio no se determinó la cantidad y periodicidad de la ingesta de las plantas mencionadas, consideramos que es necesario puntualizar en las sustancias contenidas en ellas, para poder precisar si se pueden convertir en un potencial riesgo para la madre y el niño. La supresión de alimentos principalmente de alto contenido proteico, como una forma de manejar las náuseas de la gestación, nos permite comprobar la existencia de un factor de preocupación en la medida que la embarazada no logra alimentarse adecuadamente. Estos mecanismos de autocuidados forman parte de un dispositivo sociocultural de repuesta, con ventajas de accesibilidad cultural y aun económica, que responde con mayor o menor eficacia a los problemas de salud de la mujer durante su embarazo. Es posible afirmar que la medicina doméstica, como modelo de atención a la salud de la embarazada se da en forma alternativa jugando una doble función: por un lado es una opción por sí misma, y por otro, a partir de ella es como se determinan las acciones a seguir que van desde no hacer nada, elegir una opción, hasta complementar o combinar elementos de varias opciones. Lo que nos pudiera explicar el resultado del comportamiento de los cuidados alternativos durante el embarazo ante la demanda oportuna de atención médica. Los datos obtenidos nos obligan a tener en cuenta las creencias, los conceptos y las prácticas que realizan las mujeres y, por lo tanto, a plantear la necesidad de reconocer la importancia de la participación de la medicina doméstica y tradicional en la atención a la salud de la mujer embarazada y proponer cambios en los programas educativos en salud y en la formación del recurso humano en salud, para facilitar la realización de acciones conjuntas. Consideramos como imperativo tres situacio- 
nes: (1) que se reconozca la diversidad de atención al embarazo; (2) que se acepte la medicina doméstica y tradicional como recurso a la atención del embarazo; y (3) que se trate de proponer su integración y su uso reflexivo, consciente y crítico.

\section{Referencias}

AGUIRRE, G., 1970. El Proceso de Aculturación y el Cambio Sociocultural en México. México, DF: Editorial Comunidad/Instituto de Ciencias Sociales.

ANZUREZ, C., 1983. La Medicina Tradicional en México. México, DF: UNAM/Instituto de Investigaciones Antropológicas.

BLONDELL, B. \& MARSHALL, B., 1988. Antenatal care in 20 French district risk factors and pregnancy outcome. Journal of Epidemiology and Community Health, 8:501-506.

BORDIEU, P., 1990. Sociología y Cultura. México, DF: Grijalbo.

BRUNER, J., 1988. Realidad Mental y Mundos Posibles. Barcelona: Gedisa.

CARDOSO, R., 1997. La etnicidad está en juego la ética global. In: Las Dimensiones Culturales del Cambio Global: Una Perspectiva Antropológica (L. Arispe, org.), Cuernavaca: UNAM.

GARCIA, A., 2000. Aspectos económicos de la enfermedad y la pobreza. In: La Salud en México Ante el Próximo Milenio. Memorias del XI Simposium Internacional (M. García, J. Silva \& K. Salas, org.), pp. 61-71, México, DF: Editorial Instituto Nacional de Ciencias Medicas y Nutrición Salvador Zubirán.

GERMANI, G. \& FERRERA, H., 1985. Medicina de la Comunidad. Buenos Aires: Editorial Interamericana.

HARRIS, M., 1996. Antropología Cultural. Madrid: Editorial Alianza.

INFANTE, C., 1990. Utilización de los servicios de atención prenatal: Influencia de la morbilidad percibida y de las redes sociales de ayuda. Salud Pública de México, 32:419-422.

IMSS (Instituto Mexicano del Seguro Social), 1983. Programa de Atención del Puerperio de Bajo Riesgo. México, DF: Subdirección Médica, Jefatura de Hospitales.

JARAMILLO, J. F., 1998. 101 Hierbas Medicinales. Bogotá: Editorial Martínez Roca.

KLEINBAUM, D. G. \& KUPPER, W. L., 1998. Validity general considerations. In: Epidemiology Research, Principles Methods (D. G. Kleinbaum \& E. K. Muller, ed.), pp. 175, New York: Van Nostrand Reinitold.

LANGER, A. \& LOZANO, R., 1998. La condición de la mujer y salud. In: La Condición de la Mujer en el Espacio de Salud (G. Figueroa, org.), pp. 33-77, México, DF: Colegio de México.

LARA, F. \& MARQUEZ, A., 1996. Plantas Medicinales de México: Composición, Usos y Actividad Biológica. México, DF: Universidad Autónoma de México.

LEIS, R., 1989. La Sal de los Zombies, Cultura y Educación en la Tarea Común de Despertar a los Durmientes. San José: Alforja.
LEON, N., 1905. Apuntes para la Historia de la Medicina en Michoacán. Michoacán: Instituto Médico Nacional.

LOTMAN, J. \& USPENSKIJ, B., 1979. Sobre el mecanismo semiótico de la cultura. In: Semiótica de la Cultura (J. Lotman, org.), pp. 71-82, Madrid: Ediciones Cátedra.

MARTINEZ, M., 1992. Las Plantas Medicinales de México. México, DF: Editorial Botas.

MELLADO, V.; ZOLLA, C. \& CASTAÑEDA, X., 1989. La Atención al Embarazo y Parto en el Medio Rural Mexicano. México, DF: Centro Interamericano de Estudios de Seguridad Social.

MENENDEZ, E., 1990. Autoatención y automedicación. Un sistema de transacciones permanentes. In: Antropología Médica, Orientaciones Desigualdades y Transacciones (E. Menéndez, org.), Cuadernos de la Casa Chata 179, pp. 166-203, México, DF: Centro Interamericano de Estudios Superiores de Antropología Social.

SAN MARTIN, J., 1999. Teoría de la Cultura. Madrid: Editorial Síntesis.

SSA (Secretaria de Salud), 1989. Encuesta Nacional sobre Fecundidad y Salud. México, DF: SSA.

SSA (Secretaría de Salud), 1994. Norma Oficial Mexicana para la Atención de la Mujer Durante el Embarazo, Parto y Puerperio y del Recién Nacido. México, DF: SSA.

SILVA, C., 1990. Muestreo para la Investigación en Ciencias de la Salud. Madrid: Editorial Díaz de Santos.

VILLAR, J. \& BERGSJO, P., 1997. Scientific basis for the content of routine antenatal care. I. Philosophy, recent studies, and power to eliminate or alleviate adverse maternal outcomes. Acta Obstetrica et Gynecologica Scandinavica, 76:1-14.

ZOLLA, C., 1987. Medicina tradicional y sistemas de atención a la salud. In: El Futuro de la Medicina Tradicional en Atención a la Salud en Países Latinoamericanos (X. Losoya \& C. Zolla, org.), p. 14, México, DF: Centro Interamericano de Estudios Superiores de Antropología Social.

ZOLLA, C. \& CARRILLO, A. M., 1998. Mujeres, saberes médicos e institucionalización. In: La Condición de la Mujer en el Espacio de Salud (G. Figueroa, org.), pp. 167-184, México, DF: Colegio de México.

Recibido el 11 de septiembre de 2002

Versión final presentada el 17 de marzo de 2003

Aprobado el 6 de mayo de 2003 